\title{
Commentary: Better late than later, or better safe than sorry?
}

Oz M. Shapira, MD, and Gal Aviel, MD

From the Department of Cardiothoracic Surgery, Hadassah Hebrew University Medical Center, Jerusalem, Israel. Disclosures: Authors have nothing to disclose with regard to commercial support.

Received for publication June 18, 2019; accepted for publication June 19, 2019; available ahead of print July 30, 2019.

Address for reprints: Oz M. Shapira, MD, Department of Cardiothoracic Surgery, Hadassah Hebrew University Medical Center, POB 12000, Ein Kerem Jerusalem, Israel, 91120 (E-mail: ozshapira@hadassah.org.il).

J Thorac Cardiovasc Surg 2020;159:2322-3

$0022-5223 / \$ 36.00$

Copyright (C) 2019 by The American Association for Thoracic Surgery

https://doi.org/10.1016/j.jtcvs.2019.06.049

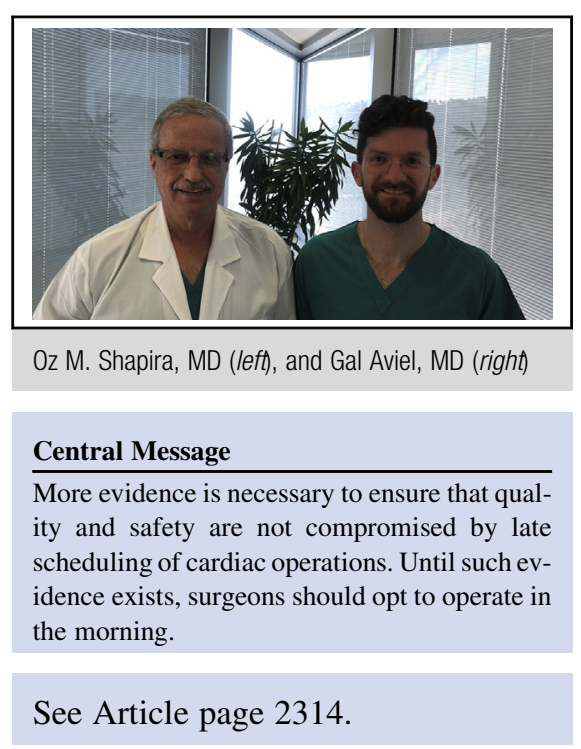

The optimal timing for scheduling an elective complex surgical procedure, such as a cardiac operation, is the morning of a regular weekday, when team members' fatigue is not a consideration, and the entire set of hospital resources are available to ensure a smooth operative course and manage unexpected perioperative adverse events. In reality, because of limited hospital resources such as operating room space and qualified personnel, this ideal may not be attainable. Rather than delaying the procedure, scheduling a cardiac operation in the afternoon becomes necessary and may be advantageous for the patient, the surgeon, and hospital administration. Shortening the time on the waiting list reduces patient anxiety and minimizes the small but definite risk of adverse cardiac events while waiting for surgery. Having a full dedicated operating room day (morning and afternoon slots) affords the surgeon with the opportunity to allocate time for administrative, academic, and educational activities. From an economic point of view, leaving an expensive resource with a huge demand-such as the operating room-empty would be extremely inefficient. All these considerations are relevant only if the quality and safety of surgery performed later in the day are not compromised (primum non nocere).

In a single-institutional study published in this issue of the Journal, Axtell and colleagues ${ }^{1}$ observed no difference in perioperative clinical outcomes and cost for elective cardiac cases that start after 3 PM. These results are encouraging, but they should be interpreted with great caution before widespread adoption.

This is a retrospective study with an inherent selection bias. The use of propensity matching methodology could not have completely eliminated bias because of hidden, nonmatched variables. In fact, only $10 \%$ of elective cases were performed in the afternoon. Clearly, the team preferred to operate in the morning. One must assume that factors not captured in the Society of Thoracic Surgeons (STS) risk-prediction models, such as frailty index and case complexity, played a role in the decision to book a case in the afternoon.

A second key issue is the size and case mix of the study population. Axtell and colleagues ${ }^{1}$ are to be congratulated for achieving outstanding clinical outcomes with a 30-day mortality of only $1 \%$. Given such a low event rate, the study cohort may have been too small to allow firm conclusions. The study cohort was low risk. The STS predicted risk of mortality (PROM) scores of morning and afternoon cases were $1.7 \%$ and $1.95 \%$, respectively. Careful look at a larger cohort consisting of intermediate-risk (STS PROM of 3\%$8 \%$ ) and high-risk (STS PROM $>8 \%$ ) patients might have yielded entirely different results.

Axtell and colleagues ${ }^{1}$ pointed to the importance of the resources available in this quaternary academic medical center, regardless of the time of day. Even in institutions with such high reliability, turnover of key members of the operating room team, such as the cardiac anesthesiologist and the scrub nurse, are more frequent and almost inevitable during afternoon hours. A recent large-scale study showed that complete handover of anesthesia care compared with no handover was associated with a significantly higher risk of all-cause mortality, hospital readmission, or major postoperative complications. ${ }^{2}$ Patients operated on late in the afternoon arrive to the intensive care unit during the evening, spending the first postoperative hours when they are most vulnerable to serious life-threatening complications during the nighttime. This may not be an important factor in a quaternary academic center, but it may be detrimental in a real-world experience in which rapid-response teams 
are less effectively used during the nighttime, leading to increased mortality as a result of failure to rescue. ${ }^{3}$

Finally, Axtell and colleagues ${ }^{1}$ used the STS 30-day quality and safety metrics to test the null hypothesis. These metrics are the best available, but by no means are they sufficient. Evaluating procedural details and reporting longterm outcomes are key to determining procedural safety, efficacy, and value. ${ }^{4}$ A prime example of this point relates to conduit selection in patients undergoing coronary artery bypass grafting. A grafting strategy of using a single internal thoracic artery and vein grafts is technically simpler, is shorter, and may be associated with fewer complications than multiple arterial grafting; however, multiple arterial grafting strategy has been shown to be associated with improved long-term survival and decreased major adverse cardiac events. ${ }^{5}$ It is only a human nature to prefer the less complex and shorter operative procedure late in the afternoon, particularly because surgeons' report cards focus on short-term outcomes and the advantages of the more complex procedure might become apparent only years later.

In summary, this study suggests that in a selected lowrisk group of patients undergoing operation in highly selected institutions, the outcomes of elective cardiac operations performed in the afternoon are equivalent to those performed in the morning. Large-scale multi-institutional studies enrolling high-risk patients and including fine procedural details and long-term outcomes are necessary to ensure that procedural quality and patient safety are not compromised by late scheduling. Until such evidence exist, surgeons should opt to be safe rather than sorry and operate in the morning.

\section{References}

1. Axtell AL, Moonsamy P, Melnitchouk S, Jassar AS, Villavicencio MA, D'alessandro da, et al. Starting elective cardiac surgery after 3 pm does not impact patient morbidity, mortality, or hospital course. J Thorac Cardiovasc Surg. 2020; 159:2314-21.e2.

2. Jones PM, Cherry RA, Allen BN, Jenkyn KM, Shariff SZ, Flier S, et al Association between handover of anesthesia care and adverse postoperative outcomes among patients undergoing major surgery. JAMA. 2018;319:143-53.

3. Churpek MM, Edelson DP, Lee JV, Carey K, Snyder A; American Heart Association's Get with the Guidelines-Resuscitation Investigators. Association between survival and time of day for rapid response team calls in a national registry. Crit Care Med. 2017:45:1677-82

4. Porter ME. What is value in health care? N Engl J Med. 2010;363:2477-81.

5. Pu A, Ding L, Shin J, Price J, Skarsgard P, Wong DR, et al. Long-term outcomes of multiple arterial coronary artery bypass grafting: a population-based study of patients in British Columbia, Canada. JAMA Cardiol. 2017;2:1187-96. 